\title{
Impact of altered growth factor signalling on endocrine response in breast cancer and the transition from ER+ to ER- disease
}

\author{
R. I. Nicholson, M. G. Giles, I. R. Hutcheson, T. Madden, J. M. W. Gee \\ Tenovus Centre for Cancer Research, Welsh School of Pharmacy, Cardiff University, Cardiff, Wales, UK.
}

\begin{abstract}
This article examines the experimental and clinical evidence of the effect of growth factor signalling in an attempt to reconcile the seeming paradox of, on the one hand, evidence that growth factor signalling can facilitate the activity of oestrogen receptor- $\alpha(E R-\alpha)$, and on the other hand, evidence that extreme growth factor signalling can promote loss of ER function and expression, thereby promoting an endocrine insensitive and ultimately ER - phenotype. The results of this analysis lead to the therapeutic possibility that ER negativity may, in some instances, be reversible to generate endocrine response and improve patient prognosis.
\end{abstract}

Keywords: Growth factors; Breast cancer, Oestrogen receptor, Endocrine resistance

\section{Introduction}

The concept that growth factor signalling can facilitate the activity of oestrogen receptor- $\alpha(E R-\alpha)$ is well established. For example, several growth factorinduced protein kinases target and phosphorylate the ER to improve its activity as a nuclear transcription factor in the presence of oestrogens and antihormonal drugs, such as tamoxifen [1]. Paradoxically, however, a literature also exists which suggests that extreme growth factor signalling can promote loss of ER function and expression, thereby promoting an endocrine insensitive and ultimately ER-phenotype. The current article presents the experimental and clinical evidence for this paradox, and addresses the therapeutic possibility that ER negativity may, in some instances, be reversible to generate endocrine response and improve patient prognosis.

Correspondence to: R. I. Nicholson, Professor of Cancer Pharmacology, Tenovus Centre for Cancer Research, Redwood Building, King Edward VII Avenue, Cardiff CF10 3XF, UK; Tel: 029 20874922; Fax: 029 20875152; E-mail: NicholsonRI@Cardiff.ac.uk

Publicaton date 27/10/04 BCO/258/2004/FO

\section{Evidence for impact of growth factor signalling on ER activity and level}

Growth factor signalling pathways positively

cross-talk with ER in a ligand-independent

manner to promote ER activity and

$E R+$ tamoxifen resistance

There is now a considerable body of literature to suggest that ER activity and transcription can be enhanced through growth factor cross-talk mechanisms. In addition to their direct stimulation of proliferation and survival signals, several growth factor- induced protein kinases, notably ERK1/2 MAPK and AKT, phosphorylate key regulatory sites on the ER protein. Many phenotypic studies in endocrine responsive clinical samples and model systems, such as MCF-7, ER + breast cancer cells indicate that prior to tamoxifen treatment, it is insulin-like growth factor (IGF)-1R signalling that enjoys close productive cross-talk with ER. Thus, IGFs activate various protein kinases that phosphorylate ER and enhance its transcriptional activity, thereby re-enforcing the actions of oestrogens to maximize growth [2]. However, our in vitro studies indicate that increased EGFR/HER2 signalling emerges during growth 
inhibition of MCF-7 by tamoxifen, and that after 3 months in culture this pathway is able to re-activate ER in a ligand-independent manner to promote acquired resistant growth. This appears to involve EGFR/HER2 priming of ERK1/2 MAPK and AKT activation, with subsequent phosphorylation on the ER AF-1 residues serine 118 and 167, promotion of ER transcriptional activity and thereby growth in the presence of antihormone. In parallel with monitoring of growth factor cross-talk effects on ER phosphorylation, there is also an emerging importance in ER+ tamoxifen-resistant cells for growth factor-induced activation of co-activators and their enhanced recruitment to the ER, including AIB1 that is promoted by HER2/ERK1/2 MAPK signalling. Co-activators are believed to promote ER transcriptional activity in several ways, modifying chromatin structure via histone acetylation (through their inherent acetyltransferase and associated histone methyltransferase activity) as well as engaging the basal transcriptional machinery. In total, increased EGFR/ HER2 growth factor signalling/ER cross-talk appears to be central in enhancing activity of the tamoxifenER complex as a nuclear transcription factor thereby promoting acquired resistant growth.

The detail of this cross-talk remains to be explored in clinical disease. However, modest increases in TGF $\alpha$, EGFR, activation of ERK1/2 MAPK, amplification of the HER2 gene and increased HER2 status are detectable when patients relapse on tamoxifen [3], and clinical benefit can be derived in acquired tamoxifen-resistant patients treated with either the anti-EGFR agent gefitinib or the HER2 antibody herceptin [1]. Moreover, while ER phosphorylation status has not to date been examined in relapse samples, ER expression is commonly retained and subsequent challenge with agents targeting $\mathrm{ER}$, such as fulvestrant, can be valuable in these acquired resistant tumours [1]. Preliminary adjuvant and neoadjuvant studies also indicate that HER2 (or EGFR) positive/ ER positive patients, while relatively resistant to tamoxifen on presentation, retain sensitivity to aromatase inhibitors [4,5]. In total, these data provide considerable clinical evidence for ER and EGFR/HER2 crosstalk contributing to ER + tamoxifen-resistant growth.

\section{More extreme or sustained growth factor signalling can adversely impact on $E R$, promoting dislocation from ER dependency, depletion of ER activity and ER loss}

While growth factors can interact positively with ER and facilitate its activity as described above, there is also literature to indicate that under more extreme (and as yet poorly-defined) conditions of growth factor signalling, dislocation from ER, blockade of
ER transcriptional activity and ER loss is possible. Thus, we have recently shown that challenge of MCF-7 cells with specific exogenous growth factors (i.e. heregulins, IGFs), or further enhancing EGFR signalling in acquired tamoxifen-resistant cells with EGF-like ligands, generates cells refractory not only to the growth-inhibitory effects of tamoxifen, but also to multiple forms of anti-ER strategies including fulvestrant and oestrogen withdrawal (i.e. 'complete endocrine insensitivity'). These observations suggest that extreme growth factor signalling can dislocate growth from ER signalling [1]. Furthermore, Stoica et al. [6-9] systematically describe the capability of exogenous challenge with EGF, IGF-1, TGF $\beta$ and TPA to downregulate ER mRNA and protein in breast cancer cells via mechanisms involving increased growth factor signalling through EGFR, IGF-1R, $\mathrm{PI} 3 \mathrm{~K} / \mathrm{AKT}, \mathrm{PKA}$ and PKCs. In the case of prolonged treatment with the PKC activator TPA, ER protein levels were reduced by $80 \%$ and there was a parallel decrease in ER mRNA, ER ligand binding, ER binding to DNA and ER/ERE activity [6]. Further agents shown to depress ER or its transcriptional activity include heregulin B1 [10,11] and retinoids [12].

Additional supportive evidence for a negative impact of exposure to extreme growth factor signalling on ER can be drawn from several stable transfection studies in ER+ breast cancer cells. Such studies demonstrate that several EGFR/HER2 signalling elements which hyper-activate ERK1/2 MAPK act to impair ER function and promote ER loss when overexpressed in ER+ breast cancer cells. In our own study, we have shown that constitutive upregulation of MEK1 in MCF-7 cells leads to a substantial increase in ERK1/2 MAPK activation, decreased ER level and marked loss of expression of the ERregulated gene progesterone receptor (McClelland et al. in preparation). Similarly, El-Ashry and colleagues [13-16] have noted precipitous falls in ER mRNA and protein following transfection of constitutively active HER2, MEK1 ( $\Delta$ mek), Raf1 ( $\Delta$ raf) or ligand-stimulated EGFR into MCF-7 cells. There is a parallel loss of oestrogen-mediated gene expression (e.g. pS2 and PR) and a marked suppression of activity of ERE-reporter gene constructs in transient transfection experiments that cannot be overcome by oestradiol treatment. Not surprisingly, the severe impact of elevated growth factor signalling on ER expression and function associated with these transfection studies result in oestrogen independence and acquisition of antioestrogen resistance. Holloway et al. [17] demonstrate that downregulation of ER by hyper-activated ERK1/2 MAPK involves the transcription factor $\mathrm{NF} K \mathrm{~B}$, which is markedly increased in activity in the various transfection models and is inhibited by abrogating ERK1/2 MAPK signalling. 
Over-expression of AKT, PKC $\alpha$ and AP-1 components have also been linked with decreased ER function, loss of ER - and endocrine-resistant states [18-20]. Interestingly, many of these parameters, including EGFR and activity of ERK1/2 MAPK and $\mathrm{NF} \kappa \mathrm{B}$, do appear to be elevated de novo in ERbreast cancer cell lines [21].

Growth factor signalling also appears to adversely impact on ER when cells are exposed in a sustained manner to the modestly elevated growth factor signalling that evolves during antihormone treatment. For example, we have recently noted that our fulvestrant-resistant cells, whose growth is promoted primarily via increased EGFR/MAP kinase signalling in the presence of minimal ER levels [22], develop a fully ER - phenotype following extended culture $(\approx 12$ months) in the presence of this pure antioestrogen. Furthermore, while early passages (3 months) of the fulvestrant-resistant sub-line were able to regain full ER signalling on removal of fulvestrant [22], the longterm resistant cells appear unresponsive to oestrogens on antioestrogen removal and ER is not recovered even after several months withdrawal. It is perhaps significant that the fulvestrant-resistant sub-line with its markedly reduced ER mRNA and ER is more susceptible to the generation of an ERphenotype than either our acquired resistant tamoxifen or oestrogen withdrawn MCF-7 variants that express considerable ER. Prolonged oestrogen deprivation has similarly been associated with evolution of an ER - phenotype from ER + T47D cells [23,24], a cell line that again has low ER levels before treatment. The resultant acquired resistant variant T47D:C4 sub-line is ER-, and expresses TGF $\alpha$ and EGFR, as well as markedly elevated PKC $\alpha$ and AP-1 activity $[23,25,26]$. It is known that chronic ER activation by oestrogen can lead to ER downregulation [24], an event involving the activated ER interacting with its own promoter to repress ER transcription [27] and also ER degradation by the ubiquitin-proteasome pathway [28]. It is thus feasible that constitutive/ chronic ER activation by growth factor signalling might similarly be able to promote such ER loss.

Interestingly, in clinical disease there is evidence that extreme growth factor signalling on presentation is prominent in ER+ endocrine-insensitive patients and particularly in ER - tumours [1]. Marked overexpression of EGFR and hyper-activation of its signalling pathway (e.g. ERK1/2 MAPK activity) have invariably been associated with ER negativity, as well as with adverse clinicopathology, metastasis and shortened relapse-free survival in breast cancer patients (reviewed in Ref. [29]). Moreover, while not as yet examined in any depth to date, sustained exposure to increased growth factor signalling may feasibly explain the phenotype of the significant cohort of patients who fail to respond to second-line endocrine challenge, where a proportion of tumours which are initially $E R+$ do lack the receptor at the time of tamoxifen relapse in the adjuvant or metastatic setting.

\section{Extreme growth factor signalling may ultimately promote silencing of ER gene expression}

Regulation of the ER gene is poorly understood and highly complex. It has multiple promoters that are regulated in a tissue-specific manner. Transacting factors for the gene include AP-2 $\gamma$ (ERF-1), oestrogen receptor promoter $B$ associated factor-1 (ERBF-1) and $\mathrm{AP}-1$, with binding sites for SP-1 and NFKB, elements that all could feasibly be influenced by growth factor signalling to positively or negatively regulate ER expression. Somewhat more is known regarding the contribution of epigenetic mechanisms in determining ER negativity in clinical breast cancers and breast cancer cell lines. DNA methylation and histone deacetylation mechanisms are believed to silence ER gene transcription in $\approx 25 \%$ of de novo ER- cancers [30]. CpG island methylation in gene promoters has been correlated with gene silencing, while histone acetylation is a crucial determinant of gene transcription. Thus, the formation of a repressor complex between DNA methyl transferase (DNMT) and histone deacetylase (HDAC) is emerging as an important mechanism in silencing the ER. While genes that are inactive may be inherently subject to such silencing mechanisms in a proportion of ER - tumours, enzymes integral to this process may again feasibly be growth factor regulated. Proof of principle is provided through studies demonstrating that pharmacological inhibition of Ras signalling is able to reverse gene methylation events in cancer models via downregulation of DNMT1 [31], while Mazumdar et al. [32] have shown that heregulin challenge induces metastasis-associated protein 1 co-repressor (MTA1) binding to ER protein to silence ERE-mediated transcriptional activity via recruitment of HDACs. Moreover, silencing of expression of the ER-regulated gene PR has been demonstrated to occur via further chromatin remodelling events following prolonged antihormonal challenge in vitro [33].

\section{Proposed model}

There remains much to learn about the role for aberrant growth factor signalling in influencing ER function and promoting ER negativity. However, we tentatively propose a model whereby increases in growth factor signalling might promote endocrine resistance 
and, in some tumours, ER negativity through a series of transitions as follows:

- Increased growth factor signalling initially induces ER phosphorylation in a ligand-independent manner and enhances co-activator recruitment to improve transcriptional activity of ER and promote $\mathrm{ER}+$ tamoxifen resistance.

- Under chronic sustained growth factor signalling conditions, cell growth is dislocated from ER, ER activity is abrogated and substantial ER loss is initiated, generating endocrine insensitivity.

- Chronic sustained growth factor signalling ultimately silences the ER gene and promotes an ER- state.

\section{Future therapeutic consequences}

While further studies are clearly required, the presented data do have exciting ramifications for possible therapeutic approaches both in ER+ endocrine- resistant and ER- disease. Manipulation of growth factor pathways (in particular blocking ERK1/2 MAPK activity) with signal transduction inhibitors would feasibly abrogate the growth factor/ ER cross-talk that appears pivotal to acquired antihormone-resistant growth. This approach could also prove valuable in ER+ endocrine insensitive disease, where growth factor signalling dislocates cell growth from ER, and may recover antihormone response in such tumours. There is in vitro data available with antigrowth factor strategies as proof of principle in both settings [1]. However, a particularly exciting consequence might be recovery of ER positivity in ER - cells, thereby restoring sensitivity to antioestrogen if used in combination. Importantly, Oh et al. [15] have demonstrated that in vitro pharmacological or dominant negative blockade of ERK1/2 MAPK signalling is able to re-instate physiological levels of ER expression and function in their stabletransfected cells, while blockade of NFKB activity using parthenolide is also valuable in this regard [17]. Of course, we must await future experimental consolidation and appropriate clinical examination, but a compelling preliminary study demonstrates that reversion of ER negativity and re-instatement of endocrine responsiveness does occur in a proportion of advanced HER2 + breast cancer patients using herceptin to inhibit growth factor signalling [34]. Although this approach may only be applicable to ER- tumours without epigenetic ER silencing, if growth factor signalling does indeed contribute to ER methylation or deacetylation then signal transduction inhibitors may also be valuable in reversing this event. Since Yang et al. [35] have shown that ER negativity in MDA-MB-231 and MDA-MB-435 cell lines can be partially reversed and some oestrogen responsiveness restored from ERE reporter constructs by the DNMT1 inhibitor aza-2-deoxycytidine (5-aza-dC) and an HDAC inhibitor trichostatin, combination of such agents with antigrowth factor strategies may prove particularly effective in ERdisease. It is envisaged that this approach could subsequently regenerate responsiveness to endocrine agents and thereby improve patient outlook.

\section{References}

1. Nicholson RI, Hutcheson IR, Knowlden JM, Jones $H$, Harper ME, Jordan N, Hiscox SE, Barrow D, Gee JM. Non-endocrine pathways and endocrine resistance: observations with antiestrogens and signal transduction inhibitors in combination. Clin Cancer Res 2004; 10: 346S-354S.

2. Yee D, Lee AV. Crosstalk between insulin-like growth factors and estrogens in breast cancer. J Mammary Gland Biol Neoplasia 2000; 5: 107-115.

3. Vogel CL, Cobleigh MA, Tripathy D, et al. Efficacy and safety of trastuzumab as a single first-line treatment of HER2-overexpressing metastic breast cancer. J Clin Oncol 2002; 20: 719-726.

4. Dowsett M, Harper-Wynne C, Boeddinghaus I, Salter J, Hills M, Dixon M, Ebbs S, Gui G, Sacks N, Smith I. HER-2 amplification impedes the antiproliferative effects of hormone therapy in estrogen receptor-positive primary breast cancer. Cancer Res 2001; 61: 8452-8458.

5. Ellis MJ, Coop A, Singh B, Mauriac L, LlombertCussac A, Janicke F, Miller WR, Evans DB, Dugan M, Brady C, Quebe-Fehling E, Borgs M. Letrozole is more effective neoadjuvant endocrine therapy than tamoxifen for ErbB-1- and/or ErbB-2-positive, estrogen receptorpositive primary breast cancer: evidence from a phase III randomized trial. J Clin Oncol 2001; 19: 3808-3816.

6. Martin MB, Garcia-Morales P, Stoica A, Solomon HB, Pierce M, Katz D, Zhang S, Danielsen M, Saceda M. Effects of 12-O-tetradecanoylphorbol-13-acetate on estrogen receptor activity in MCF-7 cells. $J$ Biol Chem 1995; 270: 25244-25251.

7. Stoica A, Saceda M, Doraiswamy VL, Coleman C, Martin MB. Regulation of estrogen receptor-alpha gene expression by epidermal growth factor. $J$ Endocrinol 2000; 165: 371-378.

8. Stoica A, Saceda M, Fakhro A, Joyner M, Martin MB. Role of insulin-like growth factor-I in regulating estrogen receptor-alpha gene expression. J Cell Biochem 2000; 76: 605-614.

9. Stoica A, Saceda M, Fakhro A, Solomon HB, Fenster BD, Martin MB. The role of transforming growth factor-beta in the regulation of estrogen receptor expression in the MCF-7 breast cancer cell line. Endocrinology 1997; 138: 1498-1505.

10. Mueller H, Kueng W, Schoumacher F, Herzer S, Eppenberger $U$. Selective regulation of steroid receptor expression in MCF-7 breast cancer cells by a novel member of the heregulin family. Biochem Biophys Res Commun 1995; 217: 1271-1278.

11. Tang CK, Perez C, Grunt T, Waibel C, Cho C, Lupu R. Involvement of heregulin-beta2 in the acquisition of the 
hormone-independent phenotype of breast cancer cells. Cancer Res 1996; 56: 3350-3358.

12. Demirpence E, Pons M, Balaguer P, Gagne D. Study of an antiestrogenic effect of retinoic acid in MCF-7 cells. Biochem Biophys Res Commun 1992; 183: 100-106.

13. El-Ashry D, Miller DL, Kharbanda S, Lippman ME, Kern FG. Constitutive Raf-1 kinase activity in breast cancer cells induces both estrogen-independent growth and apoptosis. Oncogene 1997; 15: 423-435.

14. Liu Y, El-Ashry D, Chen D, Ding IY, Kern FG. MCF-7 breast cancer cells overexpressing transfected c-erbB-2 have an in vitro growth advantage in estrogen-depleted conditions and reduced estrogen dependence and tamoxifensensitivity in vivo. Breast Cancer Res Treat 1995; 34: 97-117.

15. Oh AS, Lorant LA, Holloway JN, Miller DL, Kern FG, El-Ashry D. Hyperactivation of MAPK induces loss of ERalpha expression in breast cancer cells. Mol Endocrinol 2001; 15: 1344-1359.

16. Pietras RJ, Arboleda J, Reese DM, Wongvipat N, Pegram MD, Ramos L, Gorman CM, Parker MG, Sliwkowski MX, Slamon DJ. HER-2 tyrosine kinase pathway targets estrogen receptor and promotes hormone-independent growth in human breast cancer cells. Oncogene 1995; 10: $2435-2446$.

17. Holloway JN, Murthy S, El-Ashry D. A cytoplasmic substrate of MAPK is responsible for $\mathrm{ER} \alpha$ downregulation in breast cancer cells: the role of $\mathrm{NF}_{\kappa} \mathrm{B}$. Mol Endocrinol 2004; 18: 1396-1410.

18. Doucas V, Yaniv M. Functional interaction between estrogen receptor and proto-oncogene products C-Jun and c-Fos. CR Seances Soc Biol Fil 1991; 185: 464-474.

19. Smith LM, Wise SC, Hendricks DT, Sabichi AL, Bos T, Reddy $\mathrm{P}$, Brown $\mathrm{PH}$, Birrer MJ. cJun overexpression in MCF-7 breast cancer cells produces a tumorigenic, invasive and hormone resistant phenotype. Oncogene 1999; 18: 6063-6070.

20. Tzukerman M, Zhang XK, Pfahl M. Inhibition of estrogen receptor activity by the tumor promoter 12-O-tetradeconylphorbol-13-acetate: a molecular analysis. Mol Endocrinol 1991; 5: 1983-1992.

21. Biswas DK, Cruz AP, Gansberger E, Pardee AB. Epidermal growth factor-induced nuclear factor kappa $B$ activation: a major pathway of cell-cycle progression in estrogen-receptor negative breast cancer cells. Proc Natl Acad Sci USA 2000; 97: 8542-8547.

22. McClelland RA, Barrow D, Madden TA, Dutkowski CM, Pamment J, Knowlden JM, Gee JM, Nicholson RI. Enhanced epidermal growth factor receptor signaling in MCF7 breast cancer cells after long-term culture in the presence of the pure antiestrogen ICI 182,780 (Faslodex). Endocrinology 2001; 142: 2776-2788.

23. Murphy CS, Pink JJ, Jordan VC. Characterization of a receptor-negative, hormone-nonresponsive clone derived from a T47D human breast cancer cell line kept under estrogen-free conditions. Cancer Res 1990; 50: 72857292.
24. Pink JJ, Jordan VC. Models of estrogen receptor regulation by estrogens and antiestrogens in breast cancer cell lines. Cancer Res 1996; 56: 2321-2330.

25. Tonetti DA, Chisamore MJ, Grdina W, Schurz H, Jordan VC. Stable transfection of protein kinase $C$ alpha cDNA in hormone-dependent breast cancer cell lines. $\mathrm{Br} J$ Cancer 2000; 83: 782-791.

26. Chisamore MJ, Ahmed Y, Bentrem DJ, Jordan VC, Tonetti DA. Novel antitumor effect of estradiol in athymic mice injected with a T47D breast cancer cell line overexpressing protein kinase Calpha. Clin Cancer Res 2001; 7: 3156-3165.

27. Kaneko, KJ, Furlow JD, Gorski J. Involvement of the coding sequence for the estrogen receptor gene in autologous ligand-dependent down-regulation. Mol Endocrinol 1993; 7: 879-888.

28. Lonard DM, Nawaz Z, Smith CL, O'Malley BW. The 26S proteosome is required for estrogen receptor-alpha and coactivator turnover and efficient estrogen receptoralpha transactivation. Mol Cell 2000; 5: 939-948.

29. Gee JMW, Madden TA, Robertson JFR, Nicholson RI. Clinical response and resistance to SERMS. In: JFR Robertson, RI Nicholson, DF Hayes (Eds). Endocrine Therapy in Breast Cancer, London 2002: Martin Dunitz Ltd: 155-190.

30. Parl, FF. Multiple mechanisms of estrogen receptor gene repression contribute to ER-negative breast cancer Pharmacogenomics J 2003; 3: 251-253.

31. Alcock RA, Dey S, Chendil D, Inayat MS, Mohiuddin M, Hartman G, Chatfield LK, Gallicchio VS, Ahmed MM. Farnesyltransferase inhibitor $(\mathrm{L}-744,832)$ restores TGFbeta type II receptor expression and enhances radiation sensitivity in K-ras mutant pancreatic cancer cell line MIA PaCa-2. Oncogene 2002; 21: 7883-7890.

32. Mazumdar A, Wang RA, Mishra SK, Adam L, BagheriYarmand R, Mandal, M, Vadlamudi RK, Kumar R. Transcription repression of oestrogen receptor by metastasis-associated protein 1 corepressor. Nat Cell Biol 2001; 3: 30-37.

33. Badia E, Duchesne MJ, Semlali A, Fuentes M, Giamarchi C, Richard-Foy H, Nicolas JC Pons M. Long-term hydroxytamoxifen treatment of an MCF-7-derived breast cancer cell line irreversibly inhibits the expression of estrogenic genes through chromatin remodeling. Cancer Res 2000; 60: $4130-4138$.

34. Munzone E, Nole F, Renne G, Balduzzi A, Sanna G, Corsetto L, Goldhirsch A. Reverting estrogen receptor (ER) negative phenotype in advanced breast cancer patients over-expressing HER2 after treatment with trastuzumab plus chemotherapy. Proc Am Soc Clin Oncol 2003; 22: 848 [Abstract 3409].

35. Yan L, Nass SJ, Smith D, Nelson WG, Herman JG, Davidson NE Specific inhibition of DNMT1 by antisense oligonucleotides induces re-expression of estrogen receptor-alpha (ER) in ER-negative human breast cancer cell lines. Cancer Biol Ther 2003; 2: 552-556. 